\title{
Anomalous softening of phonon dispersion in cuprate superconductors
}

\author{
Saheli Sarkar, Maxence Grandadam, and Catherine Pépin \\ Institut de Physique Théorique, Université Paris-Saclay, CEA, CNRS, F-91191 Gif-sur-Yvette, France
}

(Received 11 September 2020; revised 31 December 2020; accepted 12 January 2021; published 18 February 2021)

\begin{abstract}
A softening of phonon dispersion has been observed experimentally in underdoped cuprate superconductors at the charge-density-wave (CDW) ordering wave vector. Interestingly, the softening occurs below the superconducting (SC) transition temperature $T_{c}$, in contrast to the metallic systems, where the softening occurs usually below the CDW onset temperature $T_{\mathrm{CDW}}$. An understanding of the "anomalous" nature of the phonon softening and its connection to the pseudogap phase in underdoped cuprates remain open questions. Employing a perturbative approach, we find that a complex interplay among the ubiquitous CDW order, SC order, and an unusually connected thermal fluctuations of these orders can explain the anomalous phonon softening below $T_{c}$. Furthermore, our formalism captures different characteristics of the low-temperature phonon softening, depending on material specificity.
\end{abstract}

DOI: 10.1103/PhysRevResearch.3.013162

The "pseudogap" phase [1-10] of the underdoped hightemperature copper-oxide-based superconductors (cuprates) remains incomprehensible even after decades of research, by and large due to a complex interplay of several symmetrybroken orders [11,12]. A universally present translational symmetry-broken order in the cuprates is a charge-densitywave (CDW) order [13-26]. Since its discovery, the CDW order has become fundamentally important due to growing evidences of its close relation to the pseudogap phase, although a full knowledge about the CDW order and its relation to the pseudogap phase remains incomplete. One leading approach to unravel the relation is to study the phonon spectrum which couples to electronic degrees of freedom, thus leaving fingerprints associated to the electronic structure.

The phonon spectrum has been largely studied in metallic systems, where the charge correlations soften the phonon spectrum, giving rise to the "Kohn anomaly" [27]. In onedimensional metals [28-30] and in some transitional-metal dichalcogenides [31], this softening grows towards zero [Fig. 1] and a full phonon softening occurs at the CDW wave vector $(Q)$ below $\mathrm{CDW}$ ordering temperature $T_{\mathrm{CDW}}$, reflecting the origin of CDW order in them. With a similar outlook, the phonon spectrum has been measured even in cuprates using different experimental techniques, like inelastic x-ray scattering [19,32-39] and inelastic neutron scattering [40,41]. All of these experiments have observed a partial phonon softening [Fig. 1] associated to $Q$ in several cuprates, only below the superconducting transition temperature $T_{c}$, in stark contrast to the metallic systems $[30,31,42,43]$. This unique occurrence of phonon softening below $T_{c}$ is hence referred to as "anomalous" phonon softening.

Published by the American Physical Society under the terms of the Creative Commons Attribution 4.0 International license. Further distribution of this work must maintain attribution to the author(s) and the published article's title, journal citation, and DOI.
In this paper we provide a theoretical explanation to the anomalous phonon softening, revealing an unusual connection between the fluctuations of CDW and superconductivity in underdoped cuprates. Though a relation between these two orders was highlighted in earlier theoretical [44-49] and experimental $[13,15,50,51]$ studies, a direct connection between their fluctuations was missing. Notably, a recent proposal [52], based on the fractionalization of a pair-density wave (PDW) order [53,54], advocates that for temperatures above $T_{c}$, a growing amount of fluctuations in CDW and superconductivity arising from a connection between them can provide a potential explanation to the pseudogap phase. Thus our work gives evidence for the fractionalization of a PDW order to be a plausible scenario for the explanation of the pseudogap phase.

In our model, we mimic the thermal fluctuations of CDW and superconductivity by introducing an inverse lifetime of quasiparticles $[55,56]$. We find that a strong phonon softening occurs only below $T_{c}$, due to quench of fluctuations in both CDW and superconductivity arising from a connection between them, as can be found in a fractionalization of a PDW order [52]. Additionally, we also show that at low temperatures, different temperature dependence of the superconducting (SC) gap and an inverse lifetime of the quasiparticle give contrasting effects on the strength of the phonon softening.

We start with a total Hamiltonian $H_{\text {tot }}$ [57], given by $H_{\text {tot }}=$ $H_{e}+H_{\mathrm{ph}}+H_{e-\mathrm{ph}}$, with

$$
\begin{aligned}
H_{e}= & \sum_{k, \sigma} \xi_{k} c_{k, \sigma}^{\dagger} c_{k, \sigma}+\sum_{k, \sigma}\left(\chi_{k} c_{k+Q, \sigma}^{\dagger} c_{k, \sigma}+\text { H.c. }\right) \\
& +\sum_{k}\left(\Delta_{k} c_{k, \uparrow}^{\dagger} c_{-k, \downarrow}^{\dagger}+\text { H.c. }\right), \\
H_{\mathrm{ph}}= & \sum_{q} \omega_{q}\left(b_{q}^{\dagger} b_{q}+b_{-q}^{\dagger} b_{-q}\right), \\
H_{e-\mathrm{ph}}= & (g / \sqrt{N}) \sum_{q} \sum_{k, \sigma}\left[c_{k+q, \sigma}^{\dagger} c_{k, \sigma}\left(b_{q}^{\dagger}+b_{-q}\right)+\text { H.c. }\right],
\end{aligned}
$$




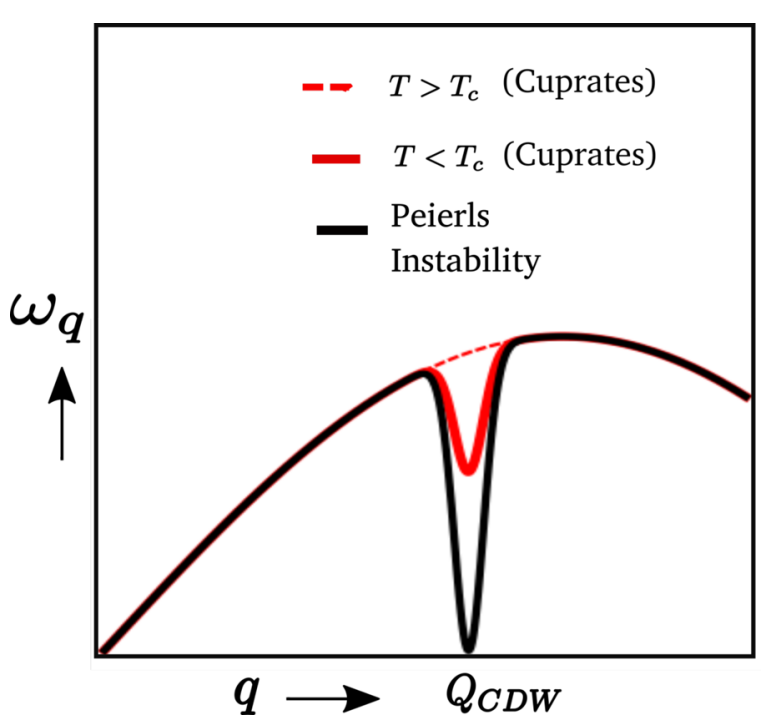

FIG. 1. Schematic representation of a full softening in metals and a partial softening in underdoped cuprates below $T_{c}$.

where $H_{e}$ is an effective mean-field Hamiltonian with SC and CDW orders. $c_{k, \sigma}^{\dagger}\left(c_{k, \sigma}\right)$ is the creation (annihilation) operator for an electron with spin $\sigma$ and momentum $k, \xi_{k}$ is the electronic dispersion, $\Delta_{k}$ is the SC order parameter, and $\chi_{k}$ is the CDW order parameter with modulation wave vector $Q . H_{\mathrm{ph}}$ is the Hamiltonian for free phonons with phonon creation operator $b_{q}^{\dagger}$ for wave-vector $q$ and frequency $\omega_{q}$. $H_{e-\text { ph }}$ is the Hamiltonian describing electron-phonon interaction with strength $g$ and $N$ is the number of lattice sites in the system. The Green's function corresponding to $H_{e}$ is given by $\hat{G}^{-1}\left(i \omega_{n}, k\right)=\left(i \omega_{n}-\hat{H}_{e}\right)$ and has a matrix form in the extended Nambu basis $\Psi_{k}^{\dagger}=\left(c_{k, \uparrow}^{\dagger}, c_{-k, \downarrow}, c_{k+Q, \uparrow}^{\dagger}, c_{-k-Q, \downarrow}\right)$, which is given by

$$
G^{-1}=\left(\begin{array}{cccc}
i \omega_{n}-\xi_{k} & -\Delta_{k} & -\chi_{k} & 0 \\
-\Delta_{k}^{*} & i \omega_{n}+\xi_{k} & 0 & \chi_{k} \\
-\chi_{k}^{*} & 0 & i \omega_{n}-\xi_{k+Q} & -\Delta_{k+Q} \\
0 & \chi_{k}^{*} & -\Delta_{k+Q}^{*} & i \omega_{n}+\xi_{k+Q}
\end{array}\right),
$$

where $\omega_{n}$ is the Matsubara frequency. We use a band structure for a prototype cuprate system [58] [see Appendix A]. Following several theoretical $[47,52,59]$ and experimental $[22,60]$ studies, we consider a CDW order parameter with $Q$ given by the axial wave vector connecting two neighboring "hot spots," the points on the Fermi surface which intersect the magnetic Brillouin zone boundary [45]. Within a mean-field treatment, the order parameter fields $\Delta_{k}$ and $\chi_{k}$ in Eq. (1) have been self-consistently obtained in Refs. [52,55] by minimizing the free energy. In this work we do not self-consistently calculate $\Delta_{k}$ and $\chi_{k}$; instead, motivated by the results of Refs. [52,55], $\chi_{k}$ is chosen such that it is maximum $\left(\chi_{\max }\right)$ is near the hot spots, falling off exponentially away from the hot spots, and $\Delta_{k}$ gaps out the rest of the Fermi surface, except for the nodal points on the Fermi surface. Away from the hot spots, we consider a $d$-wave symmetric SC gap [61] given by $\Delta_{k}=\left(\Delta_{\max } / 2\right)\left[\cos \left(k_{x}\right)-\cos \left(k_{y}\right)\right]$, where $\Delta_{\max }$ denotes the maximum gap. (a)

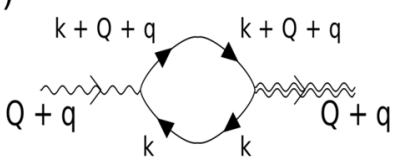

(b)

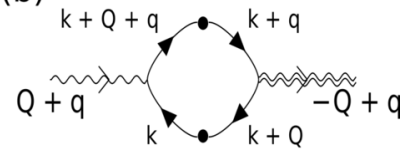

(c)

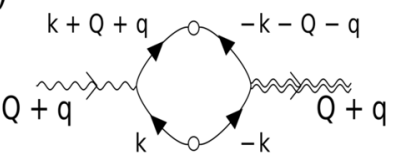

(d)

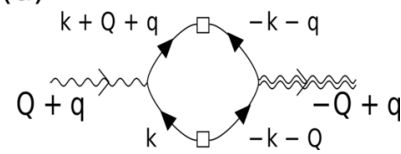

FIG. 2. (a), (b), (c), and (d) represent the Feynman diagrams for the terms in the Dyson equations [Eq. (3)] involving the self-energies $\Pi_{1}, \Pi_{3}, \Pi_{2}$, and $\Pi_{4}$, respectively, in the presence of CDW and SC orders.

The modified electronic spectrum in the presence of SC and CDW orders will renormalize the free phonon propagator, $D_{0}(z, q)=2 \omega_{q} /\left(z^{2}-\omega_{q}^{2}\right)$. To analyze this we begin by writing the imaginary time $(\tau)$ phonon propagators in matrix form in the ordered phase. The corresponding matrix elements are given by $D_{m, n}(q, \tau)=-\left\langle\mathcal{T} \phi_{q+m Q}(\tau) \phi_{q+n Q}^{\dagger}(0)\right\rangle$, where $\mathcal{T}$ is the time-ordering operator [57], $\phi_{q}$ is the phonon field operator given by $b_{q}^{\dagger}+b_{-q}$ and $m, n= \pm$. Noting that $D_{++} \equiv D_{--}:=D_{1}(z, q)$ and $D_{+-} \equiv D_{-+}:=D_{2}(z, q)$, within a perturbative treatment of electron-phonon interaction, we evaluate the renormalized phonon propagators $D_{1}$ and $D_{2}$ by using the Dyson equations

$$
\begin{aligned}
D_{1}(z, q)= & D_{0}(z, q+Q)\left[1+\Pi_{1}(z, q) D_{1}(z, q)\right. \\
& +\Pi_{2}(z, q) D_{1}(z, q)+\Pi_{3}(z, q) D_{2}(z, q) \\
& \left.+\Pi_{4}(z, q) D_{2}(z, q)\right], \\
D_{2}(z, q)= & D_{0}(z, q-Q)\left[\Pi_{1}(z, q) D_{2}(z, q)\right. \\
& +\Pi_{2}(z, q) D_{2}(z, q) \\
& \left.+\Pi_{3}(z, q) D_{1}(z, q)+\Pi_{4}(z, q) D_{1}(z, q)\right],
\end{aligned}
$$

where $\Pi_{1,2,3,4}(z, q)$ represent the phonon self-energies. The leading contributions to the Dyson equations [Eqs. (3)] are shown in Fig. 2. Explicit expressions for $\Pi_{1,2,3,4}(z, q)$ are presented in Appendix B.

We obtain the new modes for the phonon in the ordered phase by decoupling Eq. (3) with the definition $D_{ \pm}(z, q)=$ $D_{1}(z, q) \pm D_{2}(z, q)$ and then solving $D_{ \pm}(z, q)$ with the assumption that $\omega_{Q \pm q} \approx \omega_{Q}$ for small $q$. Finally, plugging in $D_{0}(z, q)$, we obtain the solutions as

$$
D_{ \pm}(z, q)=\frac{2 \omega_{Q}}{z^{2}-\omega_{Q}^{2}-2 \omega_{Q} \Pi_{ \pm}(z, q)},
$$

where $\Pi_{+}=\Pi_{1}+\Pi_{2}+\Pi_{3}+\Pi_{4}$ and $\Pi_{-}=\Pi_{1}+\Pi_{2}-$ $\Pi_{3}-\Pi_{4}$. The dispersion of the new phonon modes correspond to the values of $z$, for which denominator of Eq. (4) vanishes. Subsequently, taking only $q$ dependence in $\Pi$, the frequency for each mode is given by

$$
\Omega_{ \pm}^{2}(q)=\omega_{Q}^{2}+2 \omega_{Q} \Pi_{ \pm}(q) .
$$

These two new phonon modes in Eq. (5) with frequency $\Omega_{ \pm}$ signify branching of the free phonon near $Q$ due to presence of CDW and SC orders. The two modes with frequency $\Omega_{ \pm}$ correspond to amplitude and phase fluctuations of the CDW 


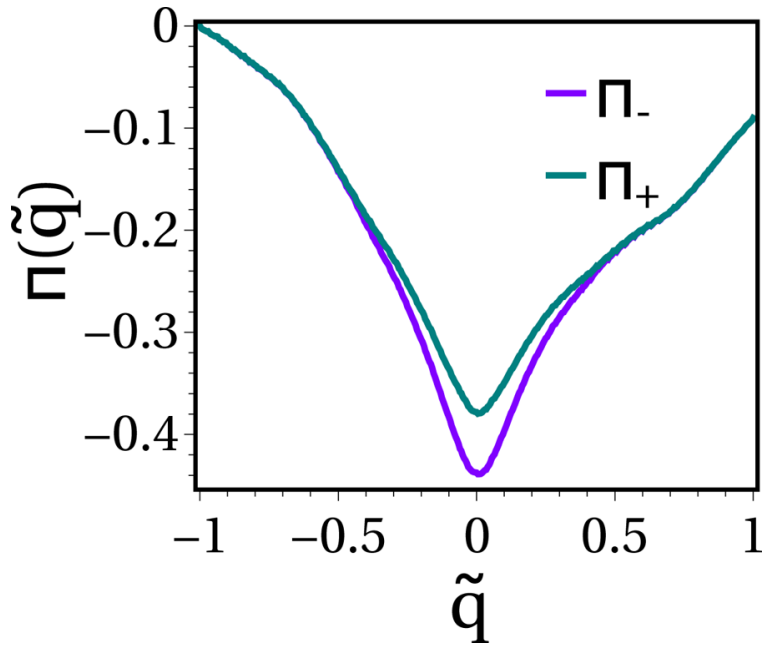

FIG. 3. Plots of the self-energy $\Pi_{ \pm}$as a function of $\tilde{q}=q-Q$ corresponding to the two renormalized phonon modes $\Omega_{ \pm}$in the presence of $\chi_{\max }=0.05$ and $\Delta_{\max }=0.05$. Both $\Pi_{ \pm}$exhibit a depletion around $\tilde{q}=0$, implying a softening in the phonon-dispersion of the two new modes $\Omega_{ \pm}$around $Q$.

order, respectively [57]. We find that the split between $\Omega_{ \pm}$ is proportional to the magnitude of the CDW order. Also, we only plot $\Pi_{ \pm}$as a function of $\tilde{q}=q-Q$, as the modes $\Omega_{ \pm}(q)$ can be easily identified from the corresponding $\Pi_{ \pm}$in Eq. (5). For depicting the strength of the phonon softening, we look at $\Pi_{ \pm}(\tilde{q})$ after subtracting $\Pi_{ \pm}(\tilde{q}=-1)$. In Fig. 3 we observe that $\Pi_{ \pm}(\tilde{q})$ decreases strongly within a finite range at around $\tilde{q}=0$, with a minimum at $\tilde{q}=0$, readily suggesting a softening of phonon frequency around $Q$. We also observe that away from $\tilde{q}=0, \Pi_{ \pm}(\tilde{q})$ goes towards zero, implying a suppression of phonon softening away from $Q$. This suggests that the effect of CDW and SC orders on the phonon are maximum at $Q$ and diminish away from it. Additionally, we notice that the suppression of $\Pi_{-}$is more than the suppression of $\Pi_{+}$and the $\tilde{q}$ dependence of $\Pi_{ \pm}$are extremely similar to each other. Hence, for a simpler presentation, in the rest of the paper we only plot $\Pi_{-}$with $\tilde{q}$ [relabeled as $\Pi(\tilde{q})$ ].

So far, we obtain a phonon softening in the presence of SC and CDW orders. However, to address the anomalous phonon softening in cuprates, we need to include fluctuation-related effects, which are major constituents governing the phase diagram of these systems. For example, such fluctuations can lead to quasiparticle scattering, which is known to have a vital role in the Fermi-arc-related physics of the pseudogap phase [62-64]. The strength of the scattering depends on temperature; while it can be large at high temperatures, a sudden reduction occurs below $T_{c}$, which can be attributed to a fractionalization of a PDW [52]. To give an idea, the proposal of fractionalization of a PDW order suggests that the fluctuation of a $\mathrm{U}(1)$ gauge field gives a constraint connecting SC and CDW fields. As a result, fractionalization of PDW occurs at an energy scale associated to the pseudogap temperature $T^{*}$; consequently, fluctuations largely increase in the system. However, below $T_{c}$ the fluctuations quench, thus yielding a global phase coherence in both CDW and SC orders and increasing the lifetime of quasiparticles.
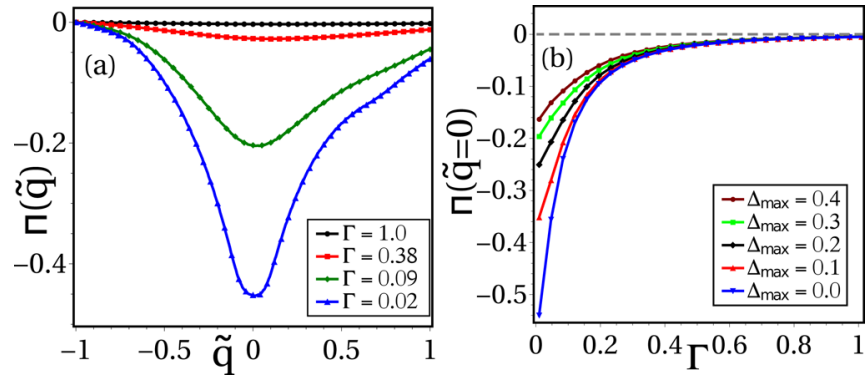

FIG. 4. (a) The variation of $\Pi(\tilde{q})$ with $\tilde{q}$ for four different values of $\Gamma$ with $\chi_{\max }=0.2$ and $\Delta_{\max }=0$. The plots portray a suppression in phonon softening with increase in $\Gamma$. (b) Plots of $\Pi(\tilde{q}=0)$ with variation in $\Gamma$ for five different values of $\Delta_{\max }$ with $\chi_{\max }=0.2$. The plots manifest a suppression in phonon softening with an increase in $\Delta_{\max }$. The effect of $\Delta_{\max }$ is strongest for low $\Gamma$ and weakest for high $\Gamma$.

In order to study the evolution of the phonon softening with temperature, we incorporate a finite inverse lifetime of quasiparticles, given by $\Gamma$, pertinent to the fluctuation-related effects in the system. The self-energy in Matsubara frequency due to $\Gamma$ can be written as $\Sigma=i \Gamma \operatorname{sgn}\left(\omega_{n}\right)$, and the Green's function in Eq. (2) will transform as

$$
G_{i, j}^{-1}\left(i \omega_{n}, k\right) \rightarrow G_{i, j}^{-1}\left(i \omega_{n}+\Sigma, k\right) .
$$

In the presence of $\Gamma$, the phonon dispersion will be modified by the real part of $\Pi(\tilde{q})$, again relabeled as $\Pi(\tilde{q})$. Detailed calculations are presented in the Appendix C.

To understand the collective effect of the SC gap and $\Gamma$ on the phonon softening, it is important to disentangle the role played by $\Gamma$ and the SC gap. Therefore we start by studying the effect of $\Gamma$, taking $\Delta_{\max }=0$. Figure 4(a) shows the variation of $\Pi(\tilde{q})$ as a function of $\tilde{q}$ for four different $\Gamma$ with $\chi_{\max }=0.2$. We notice that for very small values of $\Gamma=0.02$, there is a significantly strong phonon softening around $\tilde{q}=0$. With increasing $\Gamma$, the phonon softening starts to reduce, and for a very large $\Gamma=1.0$, the phonon softening is almost fully suppressed. We also observe that the phonon softening at $\tilde{q}=0$ is most strongly affected by $\Gamma$. Therefore, for rest of the analysis, we will concentrate on $\Pi$ at $\tilde{q}=0$ to quantify the phonon softening.

Now we inspect the role of the SC order and the interplay between superconductivity and $\Gamma$. In Fig. 4(b) we plot the variation of $\Pi(\tilde{q}=0)$ with $\Gamma$, for five different $\Delta_{\max }$ taking $\chi_{\max }=0.2$. We notice that $\Delta_{\max }$ has a prominent effect when $\Gamma$ is very small, as can be seen from the change in $\Pi(\tilde{q}=0)$ at around $\Gamma \sim 0.05$. In this regime, $\Delta_{\max }$ weakens the softening of phonon. A similar effect on phonons in the SC phase has been indicated in conventional $s$-wave superconductors $[65,66]$. With increasing $\Gamma$, for example, at around $\Gamma \sim 0.3$, the effect of $\Delta_{\max }$ becomes less significant. Finally, for very large $\Gamma \simeq 1.0$, changing $\Delta_{\max }$ has almost no effect. These results highlight two crucial points. First, both superconductivity and $\Gamma$ suppress the phonon softening. Second, the role of $\Delta_{\max }$ is prominent at low $\Gamma$ while negligible for large $\Gamma$.

We have seen that the introduction of superconductivity suppresses the phonon softening, while experiments observe a seemingly opposite characteristic of enhancement of phonon 

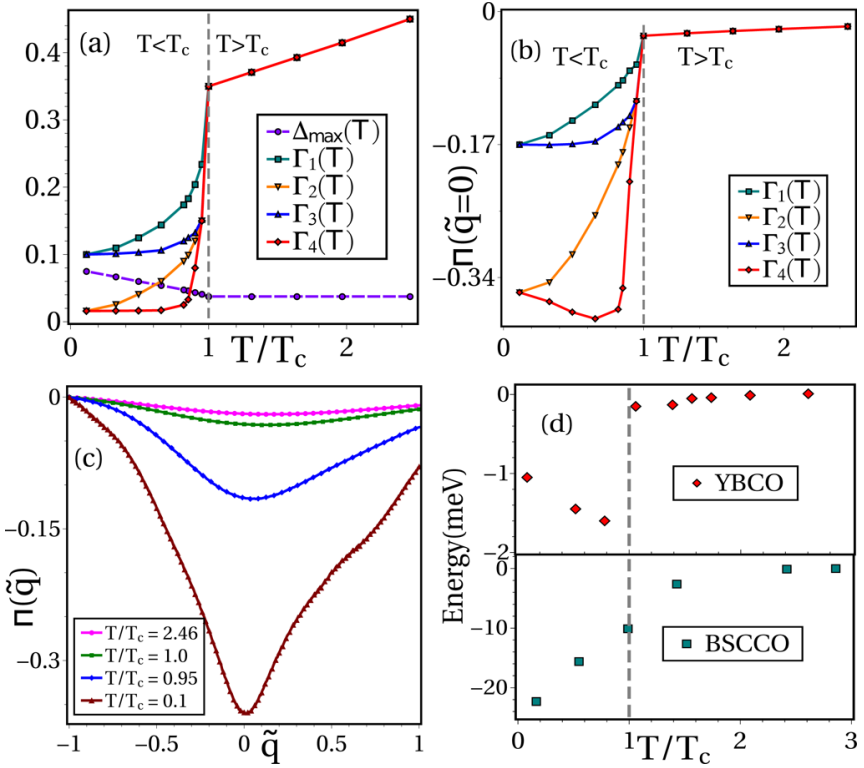

FIG. 5. (a) Different sets of $T$ dependence for inverse lifetime of quasiparticles denoted by $\Gamma_{1}, \Gamma_{2}, \Gamma_{3}$, and $\Gamma_{4}$. The $T$ dependence of the SC gap is denoted by $\Delta_{\max }(T)$. In all cases, $\chi_{\max }=\Delta_{\max }$. (b) The $T$ dependence of $\Pi(\tilde{q}=0)$ for different parameter sets in (a). A large negative value of $\Pi(\tilde{q}=0)$ in the regime $T \lesssim T_{c}$ implies a strong enhancement of phonon softening, while $\Pi(\tilde{q}=0) \rightarrow 0$ implies a strong suppression in phonon softening in the regime $T>T_{c}$. (c) The variation of $\Pi(\tilde{q})$ with $\tilde{q}$ at four different temperatures for parameter set $\Gamma_{4}$ and $\Delta_{\max }(T)$ shown in (a). (d) Schematic representation of the experimental results of phonon softening at CDW wave vector for YBCO and BSCCO, adopted from Refs. [32,34].

softening below $T_{c}$. At this point, we should also notice that $\Gamma$ suppresses the phonon softening, as shown in Fig. 4(a). Moreover, $\Gamma$ is expected to increase with temperature due to increase in fluctuations, whereas $\Delta_{\max }$ is expected to decrease with temperature, for example, in a simple BCS-type scenario. Thus they behave in opposite manner with temperature.

We consider temperature $(T)$ dependence phenomenologically in $\Delta_{\max }$ and $\Gamma$, similar to the $T$ dependence used in explaining spectral function in angle-resolved photoelectron spectroscopy (ARPES) experiments [56]. The $T$ dependence of $\Delta_{\max }$ and $\Gamma$ are shown in Fig. 5(a). Below $T_{c}, \Delta_{\max }$ decreases with $T$, remaining approximately constant above $T_{c}$. Here we also consider $\chi_{\max }$ to be equal to $\Delta_{\max }$. This is motivated by Raman spectroscopy measurements, which observe the maximum of the gaps in particle-particle $\left(\Delta_{\max }\right)$ and particle-hole $\left(\chi_{\max }\right)$ channels to be equal in a varied range of doping [50] and also in different cuprate families [67]. To illustrate how different $T$ dependence of $\Gamma$ and $\Delta_{\max }$ can give different features in phonon softening, we use four different types of $T$ dependence for $\Gamma$, denoted by $\Gamma_{1}, \Gamma_{2}, \Gamma_{3}$, and $\Gamma_{4}$ in Fig. 5(a). Note that they differ in magnitudes compared to $\Delta_{\text {max }}$. In all these cases, $\Gamma$ reduces significantly below $T_{c}$, with the strongest fall in $\Gamma_{4}$ and the weakest fall in $\Gamma_{1}$, but still remains finite even in the limit $T \rightarrow 0$ [68]. The strong reduction of $\Gamma$ below $T_{c}$ is a manifestation of the fact that not only fluctuation in superconductivity but also the CDW fluctuation simultaneously quench below this temperature. Moreover, we considered in all the cases, a linear $T$ dependence for $\Gamma$ for $T>T_{c}$, as suggested in some earlier works [69,70].

In Fig. 5(b) we plot $\Pi(\tilde{q}=0)$ for the parameters in Fig. 5(a). We start by closely inspecting the $\Gamma_{4}$ case in Fig. 5(b). We observe that the values of $\Pi(\tilde{q}=0)$ are close to zero for high temperatures $\left(T \gg T_{c}\right)$, implying that the phonon softening is strongly suppressed. Remarkably, we observe that for temperatures $T \lesssim T_{c}$, the values of $\Pi(\tilde{q}=0)$ reduce sharply towards more negative values, which suggests that the phonon softening enhances strongly. But surprisingly, towards further lower temperatures below $T_{c}, \Pi(\tilde{q}=0)$ enhances, which implies a suppression in phonon softening. However, the phonon softening below $T_{c}$ always remains stronger as compared to $T>T_{c}$. Very similar features have been observed in $\mathrm{YBa}_{2} \mathrm{Cu}_{3} \mathrm{O}_{6+y}$ (YBCO) [32], as shown schematically in Fig. 5(d). In Fig. 5(c) we present the full $\tilde{q}$ dependence of $\Pi$ at four different temperatures for the case $\Gamma_{4}$. We observe that away from $\tilde{q}=0$, phonon softening is less sensitive to the variation of temperature. A similar feature has been found in experiments [32,34].

Next we closely investigate the $\Gamma_{1}$ case in Fig. 5(b) for $T \lesssim T_{c}$. Very interestingly, the features for $T \lesssim T_{c}$ possess marked differences from the $\Gamma_{4}$ case. We notice a smoother enhancement in phonon softening just below $T_{c}\left(T \sim T_{c}\right)$, while the enhancement is more rapid and sharper for the $\Gamma_{4}$ case. In particular, towards lower temperatures $(\mathrm{T} \rightarrow 0)$, a further enhancement in phonon softening can be noticed in contrast to the suppression observed for $\Gamma_{4}$. Analogous features in phonon softening have been also observed in $\mathrm{Bi}_{2} \mathrm{Sr}_{2} \mathrm{CaCu}_{2} \mathrm{O}_{8+y}$ (BSCCO) [34], schematically presented in Fig. 5(d). To demonstrate the different features in phonon softening resulting from an intricate interplay between SC gap and $\Gamma$ below $T_{c}$, we plot results for two more cases $\Gamma_{2}$ and $\Gamma_{3}$, shown in Fig. 5(b). Below $T_{c}$, for $\Gamma_{2}$, phonon softening sharply enhances as compared to the case for $\Gamma_{3}$ as $T \rightarrow 0$.

We would like to point out that in lanthanum (La)based cuprates like $\mathrm{La}_{1.875} \mathrm{Ba}_{0.125} \mathrm{CuO}_{4}$ (LBCO) [33] and $\mathrm{La}_{2-x} \mathrm{Sr}_{x} \mathrm{CuO}_{4+\delta}$ (LSCO) [71], a phonon softening of similar order of magnitude to that of YBCO has been observed near $T_{c}$. However, the temperature dependence of the phonon softening in these two families of cuprates exhibits different behaviors above $T_{c}$. In the case of YBCO, the softening diminishes above $T_{c}$, while for LSCO the strength of the softening at a temperature much higher than $T_{c}$ remains almost similar to the strength observed at $T_{c}$. This difference might be due to varied CDW phenomenology [72] in these two families of cuprates. While CDW possesses a checkerboard pattern in the case of $\mathrm{YBCO}$, the $\mathrm{CDW}$ has a stripy nature in La-based cuprates. Moreover, presence of an incommensurate magnetic order associated with the stripe formation brings in further complexity in La-based cuprates. Therefore additional ingredients, which are not incorporated in this work, are required to fully understand the nature of phonon softening in La-based cuprates.

In summary, within a mean-field description of superconductivity and charge-density wave (CDW), describing underdoped cuprates, we obtained a softening of the phonon dispersion associated to the CDW wave vector $(Q)$. The crucial finding of our work is that only reduced amount of fluctuations in both CDW and superconducting (SC) orders 


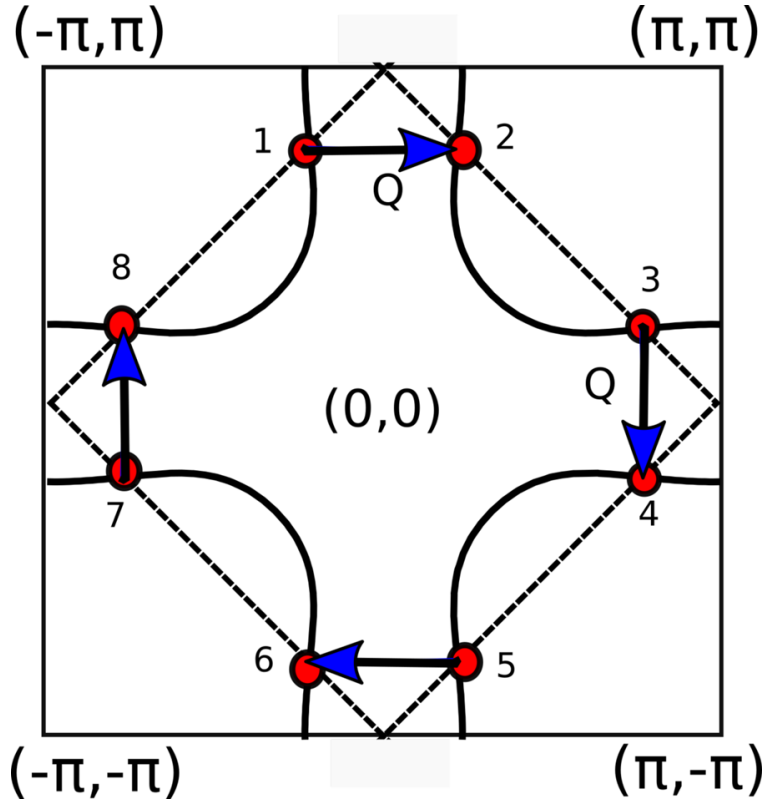

FIG. 6. Fermi surface for a prototype cuprate band structure. The solid black curves represent the Fermis surface associated to the dispersion $\xi_{k}$. The dashed black lines represent the magnetic Brillouin zone boundary of the system. The intersection of the magnetic Brillouin zone boundary and the Fermi surface are marked by red dots, representing the hot spots on the Fermi surface. The CDW modulation wave vector $Q$, indicated by the arrows, is considered to be parallel to the crystallographic axes as shown in the figure.

below $T_{c}$, can describe the "anomalous" phonon softening. A reduction in the fluctuations of $\mathrm{SC}$ and $\mathrm{CDW}$ orders below $T_{c}$ can occur due to the fractionalization of a PDW order [52]. Thus the anomalous softening gives a unique signature towards the fractionalization phenomenon. Moreover, we also found that the features of phonon softening at low temperatures depend on an intricate interplay between SC order and fluctuations. In this work we considered the strength of electron-phonon coupling to be momentum $(k)$ independent. However, the formalism in this work can easily be extended to include $k$-dependent electron-phonon coupling. We expect, in such a scenario, that phonon softening will still occur at $Q$ only below $T_{c}$, but the softening will have a different wave-vector dependence around $Q$. We believe our results can find applications in many two-dimensional materials where an interplay between CDW and SC orders plays an important role, thus opening much broader prospects of our work.

We acknowledge A. Banerjee and Y. Sidis for valuable discussions. This work has received financial support from the ERC under Grant Agreement No. AdG-694651CHAMPAGNE.

\section{APPENDIX A: THE MODEL AND PARAMETERS}

In this Appendix, we discuss the model describing the cuprate in the presence of charge-density-wave (CDW) and superconducting (SC) orders. To analyze the phonon dispersion in the presence of $\mathrm{CDW}$ and SC orders, we start with a total Hamiltonian $H_{\text {tot }}$, which incorporates an effective mean-field electronic Hamiltonian $\left(H_{e}\right)$ describing CDW and $\mathrm{SC}$ orders, the Hamiltonian for free phonons $\left(H_{\mathrm{ph}}\right)$, and the electron-phonon interaction Hamiltonian $\left(H_{e-\mathrm{ph}}\right)$ as given in Eq. (1). The inverse Green's function matrix $\hat{G}^{-1}\left(i \omega_{n}, k\right)=$ $\left(i \omega_{n}-\hat{H}_{e}\right)$ corresponding to the Hamiltonian $H_{e}$ in the extended Nambu basis $\Psi_{k}^{\dagger}=\left(c_{k, \uparrow}^{\dagger}, c_{-k, \downarrow}, c_{k+Q, \uparrow}^{\dagger}, c_{-k-Q, \downarrow}\right)$ is given by

$$
G^{-1}\left(i \omega_{n}, k\right)=\left(\begin{array}{cccc}
i \omega_{n}-\xi_{k} & -\Delta_{k} & -\chi_{k} & 0 \\
-\Delta_{k}^{*} & i \omega_{n}+\xi_{k} & 0 & \chi_{k} \\
-\chi_{k}^{*} & 0 & i \omega_{n}-\xi_{k+Q} & -\Delta_{k+Q} \\
0 & \chi_{k}^{*} & -\Delta_{k+Q}^{*} & i \omega_{n}+\xi_{k+Q}
\end{array}\right),
$$

where $\xi_{k}$ is the electronic dispersion, given by $\xi_{k}=$ $2 t_{1}\left[\cos k_{x}+\cos k_{y}\right]+4 t_{2} \cos \left(k_{x}\right) \cos \left(k_{y}\right)+2 t_{3}\left[\cos \left(2 k_{x}\right)+\cos \right.$ $\left.\left(2 k_{y}\right)\right]-\mu$, with $t_{1}=-70.25 \mathrm{meV}, t_{2}=34.75 \mathrm{meV}$, $t_{3}=-11 \mathrm{meV}$, and $\mu=-89 \mathrm{meV}$. In this paper all energy scales are expressed in units of $t_{1} . \Delta_{k}$ is the SC order parameter, and $\chi_{k}$ is the CDW order parameter with the finite wave vector $Q . \omega_{n}$ is the Matsubara frequency. $\Psi$ is the Nambu spinor, $c_{k, \uparrow}^{\dagger}$ is the creation operator for an electronic state with wave vector $k$ and up spin, and $c_{-k, \downarrow}$ is the annihilation operator for an electronic state with wave vector $-k$ and down spin. The Fermi surface for the electronic dispersion $\xi_{k}$, hot spots, and the CDW wave vectors $(Q)$ parallel to the crystallographic axes are shown in Fig. 6.

\section{APPENDIX B: DYSON EQUATIONS AND CALCULATION OF THE SELF-ENERGY $\Pi$}

In this Appendix, we present the derivation of the phonon propagators, renormalized due to CDW and SC orders. The free phonon is given by the propagator $D_{0}(z, q)=2 \omega_{q} /\left(z^{2}-\omega_{q}^{2}\right)$, where $\omega_{q}$ is the frequency of the phonon mode for wave vector $q$ and $z$ is a complex frequency ( $\operatorname{Im} z>0$ ). The CDW and SC orders will couple to the free phonon, modifying the propagator, which will consequently give rise to phonon modes with renormalized dispersion. To evaluate the renormalized dispersions, we start with Matsubara phonon propagator in matrix form whose elements are given by $D_{m, n}(q, \tau)=-\left\langle\mathcal{T} \phi_{q+m Q}(\tau) \phi_{q+n Q}^{\dagger}(0)\right\rangle$, where $\mathcal{T}$ is the time-ordering operator, and $m, n= \pm$. By noting that $D_{++} \equiv D_{--}:=D_{1}(z, q)$ and $D_{+-} \equiv D_{-+}:=D_{2}(z, q)$, within a perturbative approach for the electron-phonon interaction in Hamiltonian of Eq. (1), the Dyson's equations involving self-energies in the presence of SC and CDW orders will give the modified phonon propagators $D_{1}, D_{2}$ and can be written as

$$
\begin{aligned}
& D_{1}(z, q)=D_{0}(z, q+Q)\left[1+\Pi_{1}(z, q) D_{1}(z, q)+\Pi_{1}(z, q) D_{1}(z, q)+\Pi_{3}(z, q) D_{2}(z, q)+\Pi_{4}(z, q) D_{2}(z, q)\right] \\
& D_{2}(z, q)=D_{0}(z, q-Q)\left[\Pi_{5}(z, q) D_{2}(z, q)+\Pi_{6}(z, q) D_{2}(z, q)+\Pi_{7}(z, q) D_{1}(z, q)+\Pi_{8}(z, q) D_{1}(z, q)\right] .
\end{aligned}
$$


The self-energies $\Pi_{1}, \Pi_{2}, \Pi_{3}, \Pi_{4}, \Pi_{5}, \Pi_{6}, \Pi_{7}$, and $\Pi_{8}$ in Eq. (B1) are given by

$$
\begin{aligned}
& \Pi_{1}(\omega, q)=\frac{g^{2}}{N} \sum_{k, i \omega_{n}}\left[G_{11}\left(k, i \omega_{n}\right) G_{33}\left(k+q, i \omega_{n}+i \epsilon_{n}\right)+(k \rightarrow k-q)\right] \\
& \Pi_{2}(\omega, q)=\frac{g^{2}}{N} \sum_{k, i \omega_{n}}\left[G_{12}\left(k, i \omega_{n}\right) G_{34}\left(k+q, i \omega_{n}+i \epsilon_{n}\right)+(k \rightarrow k-q)\right] \\
& \Pi_{3}(\omega, q)=\frac{g^{2}}{N} \sum_{k, i \omega_{n}}\left[G_{13}\left(k, i \omega_{n}\right) G_{31}\left(k+q, i \omega_{n}+i \epsilon_{n}\right)+(k \rightarrow k-q)\right] \\
& \Pi_{4}(\omega, q)=\frac{g^{2}}{N} \sum_{k, i \omega_{n}}\left[G_{14}\left(k, i \omega_{n}\right) G_{32}\left(k+q, i \omega_{n}+i \epsilon_{n}\right)+(k \rightarrow k-q)\right] \\
& \Pi_{5}(\omega, q)=\frac{g^{2}}{N} \sum_{k, i \omega_{n}}\left[G_{33}\left(k, i \omega_{n}\right) G_{11}\left(k+q, i \omega_{n}+i \epsilon_{n}\right)+(k \rightarrow k-q)\right] \\
& \Pi_{6}(\omega, q)=\frac{g^{2}}{N} \sum_{k, i \omega_{n}}\left[G_{34}\left(k, i \omega_{n}\right) G_{12}\left(k+q, i \omega_{n}+i \epsilon_{n}\right)+(k \rightarrow k-q)\right] \\
& \Pi_{7}(\omega, q)=\frac{g^{2}}{N} \sum_{k, i \omega_{n}}\left[G_{31}\left(k, i \omega_{n}\right) G_{13}\left(k+q, i \omega_{n}+i \epsilon_{n}\right)+(k \rightarrow k-q)\right] \\
& \Pi_{8}(\omega, q)=\frac{g^{2}}{N} \sum_{k, i \omega_{n}}\left[G_{32}\left(k, i \omega_{n}\right) G_{14}\left(k+q, i \omega_{n}+i \epsilon_{n}\right)+(k \rightarrow k-q)\right] .
\end{aligned}
$$

With a further assumption of small $q$, we note that $\Pi_{1} \approx \Pi_{5}, \Pi_{2} \approx \Pi_{6}, \Pi_{3} \approx \Pi_{7}$, and $\Pi_{4} \approx \Pi_{8}$, which gives the final form of the Dyson's equations as

$$
\begin{aligned}
& D_{1}(z, q)=D_{0}(z, q+Q)\left[1+\Pi_{1}(z, q) D_{1}(z, q)+\Pi_{2}(z, q) D_{1}(z, q)+\Pi_{3}(z, q) D_{2}(z, q)+\Pi_{4}(z, q) D_{2}(z, q)\right], \\
& D_{2}(z, q)=D_{0}(z, q-Q)\left[\Pi_{1}(z, q) D_{2}(z, q)+\Pi_{2}(z, q) D_{2}(z, q)+\Pi_{3}(z, q) D_{1}(z, q)+\Pi_{4}(z, q) D_{1}(z, q)\right] .
\end{aligned}
$$

The corresponding Feynman diagrams for the above self-energies in Eq. (B3) are shown in Fig. 2. In Eq. (B3) we consider the strength of electron-phonon interaction, $g$ to be $k$ independent, and the number of lattice sites in the system to be $N$. The Dyson's equations from $D_{1}$ and $D_{2}$ can be decoupled to obtain the new renormalized phonon modes by introducing $D_{ \pm}(z, q)=$ $D_{1}(z, q) \pm D_{2}(z, q)$ and then solving for $D_{ \pm}$. The solution for the frequencies of the new phonon modes are

$$
\Omega_{ \pm}^{2}(q)=\omega_{Q}^{2}+2 \omega_{Q} \Pi_{ \pm}(q)
$$

where $\Omega_{ \pm}(q)$ represents the renormalized frequencies [also given in Eq. (5)], and $\Pi_{+}=\Pi_{1}+\Pi_{2}+\Pi_{3}+\Pi_{4}$ and $\Pi_{-}=\Pi_{1}+$ $\Pi_{2}-\Pi_{3}-\Pi_{4}$. The Green's function matrix elements $[G(i, j)]$ that are appearing in the self-energy expressions in Eq. (B3) are given by

$$
\begin{aligned}
& G_{11}\left(k, i \omega_{n}\right)=\frac{A_{1}}{\left(i \omega_{n}+E_{k}^{-}\right)}+\frac{A_{2}}{\left(E_{k}^{-}-i \omega_{n}\right)}+\frac{A_{3}}{\left(E_{k}^{+}+i \omega_{n}\right)}+\frac{A_{4}}{\left(E_{k}^{+}-i \omega_{n}\right)}, \\
& G_{33}\left(k, i \omega_{n}\right)=\frac{A_{5}}{\left(i \omega_{n}+E_{k}^{-}\right)}+\frac{A_{6}}{\left(E_{k}^{-}-i \omega_{n}\right)}+\frac{A_{7}}{\left(E_{k}^{+}+i \omega_{n}\right)}+\frac{A_{8}}{\left(E_{k}^{+}-i \omega_{n}\right)}, \\
& G_{12}\left(k, i \omega_{n}\right)=\frac{A_{9}}{\left(i \omega_{n}+E_{k}^{-}\right)}+\frac{A_{10}}{\left(E_{k}^{-}-i \omega_{n}\right)}+\frac{A_{11}}{\left(E_{k}^{+}+i \omega_{n}\right)}+\frac{A_{12}}{\left(E_{k}^{+}-i \omega_{n}\right)}, \\
& G_{34}\left(k, i \omega_{n}\right)=\frac{A_{13}}{\left(i \omega_{n}+E_{k}^{-}\right)}+\frac{A_{14}}{\left(E_{k}^{-}-i \omega_{n}\right)}+\frac{A_{15}}{\left(E_{k}^{+}+i \omega_{n}\right)}+\frac{A_{16}}{\left(E_{k}^{+}-i \omega_{n}\right)}, \\
& G_{13}\left(k, i \omega_{n}\right)=\frac{A_{17}}{\left(i \omega_{n}+E_{k}^{-}\right)}+\frac{A_{18}}{\left(E_{k}^{-}-i \omega_{n}\right)}+\frac{A_{19}}{\left(E_{k}^{+}+i \omega_{n}\right)}+\frac{A_{20}}{\left(E_{k}^{+}-i \omega_{n}\right)}, \\
& G_{31}\left(k, i \omega_{n}\right)=\frac{A_{21}}{\left(i \omega_{n}+E_{k}^{-}\right)}+\frac{A_{22}}{\left(E_{k}^{-}-i \omega_{n}\right)}+\frac{A_{23}}{\left(E_{k}^{+}+i \omega_{n}\right)}+\frac{A_{24}}{\left(E_{k}^{+}-i \omega_{n}\right)}, \\
& G_{14}\left(k, i \omega_{n}\right)=\frac{A_{25}}{\left(i \omega_{n}+E_{k}^{-}\right)}+\frac{A_{26}}{\left(E_{k}^{-}-i \omega_{n}\right)}+\frac{A_{27}}{\left(E_{k}^{+}+i \omega_{n}\right)}+\frac{A_{28}}{\left(E_{k}^{+}-i \omega_{n}\right)},
\end{aligned}
$$




$$
G_{32}\left(k, i \omega_{n}\right)=\frac{A_{29}}{\left(i \omega_{n}+E_{k}^{-}\right)}+\frac{A_{30}}{\left(E_{k}^{-}-i \omega_{n}\right)}+\frac{A_{31}}{\left(E_{k}^{+}+i \omega_{n}\right)}+\frac{A_{32}}{\left(E_{k}^{+}-i \omega_{n}\right)},
$$

where $E_{k}^{ \pm}$is the renormalized electronic dispersion and is given by

$$
E_{k}^{ \pm}= \pm \frac{1}{\sqrt{2}} \sqrt{\beta_{k}^{2}-\eta_{k}}
$$

with

$$
\begin{aligned}
\beta_{k}^{2} & =E_{1 k}^{2}+E_{2 k}^{2}+\Delta_{1 k}^{2}+\Delta_{2 k}^{2}+2 \chi_{k}^{2}, \\
\eta_{k}^{2} & =\left[\left(E_{1 k}+E_{2 k}\right)\left(E_{1 k}-E_{2 k}\right)+\left(\Delta_{1 k}+\Delta_{2 k}\right)\left(\Delta_{1 k}-\Delta_{2 k}\right)\right]^{2}+4 \chi_{k}^{2}\left[\left(E_{1 k}+E_{2 k}\right)^{2}+\left(\Delta_{1 k}-\Delta_{2 k}\right)^{2}\right],
\end{aligned}
$$

where we use the following simplified notations: $\xi_{k}=E_{1 k}, \xi_{k+Q}=E_{2 k}, \Delta_{k}=\Delta_{1 k}$, and $\Delta_{k+Q}=\Delta_{2 k}$. In the right-hand side of the Eq. (B5), the numerators are given by

$$
\begin{aligned}
& A_{1}=\frac{\left(E_{1 k}-E_{k}^{-}\right)\left(E_{2 k}^{2}-\left(E_{k}^{-}\right)^{2}+\Delta_{2 k}^{2}\right)-\left(E_{2 k}+E_{k}^{-}\right) \chi_{k}^{2}}{2 E_{k}^{-}\left(E_{k}^{-}-E_{k}^{+}\right)\left(E_{k}^{-}+E_{k}^{+}\right)}, \\
& A_{2}=\frac{\left(E_{1 k}+E_{k}^{-}\right)\left(E_{2 k}^{2}-\left(E_{k}^{-}\right)^{2}+\Delta_{2 k}^{2}\right)+\left(-E_{2 k}+E_{k}^{-}\right) \chi_{k}^{2}}{2 E_{k}^{-}\left(E_{k}^{-}-E_{k}^{+}\right)\left(E_{k}^{-}+E_{k}^{+}\right)} \text {, } \\
& A_{3}=\frac{-\left(E_{1 k}-E_{k}^{+}\right)\left(E_{2 k}^{2}-\left(E_{k}^{+}\right)^{2}+\Delta_{2 k}^{2}\right)+\left(E_{2 k}+E_{k}^{+}\right) \chi_{k}^{2}}{2 E_{k}^{+}\left(E_{k}^{-}-E_{k}^{+}\right)\left(E_{k}^{-}+E_{k}^{+}\right)}, \\
& A_{4}=\frac{-\left(E_{1 k}+E_{k}^{+}\right)\left(E_{2 k}^{2}-\left(E_{k}^{+}\right)^{2}+\Delta_{2 k}^{2}\right)+\left(E_{2 k}-E_{k}^{+}\right) \chi_{k}^{2}}{2 E_{k}^{+}\left(E_{k}^{-}-E_{k}^{+}\right)\left(E_{k}^{-}+E_{k}^{+}\right)}, \\
& A_{5}=\frac{\left(E_{2 k}-E_{k}^{-}\right)\left(E_{1 k}^{2}-\left(E_{k}^{-}\right)^{2}+\Delta_{1 k}^{2}\right)-\left(E_{1 k}+E_{k}^{-}\right) \chi_{k}^{2}}{2 E_{k}^{-}\left(E_{k}^{-}-E_{k}^{+}\right)\left(E_{k}^{-}+E_{k}^{+}\right)}, \\
& A_{6}=\frac{\left(E_{2 k}+E_{k}^{-}\right)\left(E_{1 k}^{2}-\left(E_{k}^{-}\right)^{2}+\Delta_{1 k}^{2}\right)+\left(-E_{1 k}+E_{k}^{-}\right) \chi_{k}^{2}}{2 E_{k}^{-}\left(E_{k}^{-}-E_{k}^{+}\right)\left(E_{k}^{-}+E_{k}^{+}\right)}, \\
& A_{7}=\frac{-\left(E_{2 k}-E_{k}^{+}\right)\left(E_{1 k}^{2}-\left(E_{k}^{+}\right)^{2}+\Delta_{1 k}^{2}\right)+\left(E_{1 k}+E_{k}^{+}\right) \chi_{k}^{2}}{2 E_{k}^{+}\left(E_{k}^{-}-E_{k}^{+}\right)\left(E_{k}^{-}+E_{k}^{+}\right)} \\
& A_{8}=\frac{-\left(E_{2 k}+E_{k}^{+}\right)\left(E_{1 k}^{2}-\left(E_{k}^{+}\right)^{2}+\Delta_{1 k}^{2}\right)+\left(E_{1 k}-E_{k}^{+}\right) \chi_{k}^{2}}{2 E_{k}^{+}\left(E_{k}^{-}-E_{k}^{+}\right)\left(E_{k}^{-}+E_{k}^{+}\right)}, \\
& A_{9}=\frac{\Delta_{1 k}\left(E_{2 k}^{2}-\left(E_{k}^{-}\right)^{2}+\Delta_{2 k}^{2}\right)+\Delta_{2 k} \chi_{k}^{2}}{2 E_{k}^{-}\left(E_{k}^{-}-E_{k}^{+}\right)\left(E_{k}^{-}+E_{k}^{+}\right)}, \\
& A_{10}=\frac{\Delta_{1 k}\left(E_{2 k}^{2}-\left(E_{k}^{-}\right)^{2}+\Delta_{2 k}^{2}\right)+\Delta_{2 k} \chi_{k}^{2}}{2 E_{k}^{-}\left(E_{k}^{-}-E_{k}^{+}\right)\left(E_{k}^{-}+E_{k}^{+}\right)}, \\
& A_{11}=(-1) \frac{\Delta_{1 k}\left(E_{2 k}^{2}-\left(E_{k}^{+}\right)^{2}+\Delta_{2 k}^{2}\right)+\left(\Delta_{2 k}\right) \chi_{k}^{2}}{2 E_{k}^{+}\left(E_{k}^{-}-E_{k}^{+}\right)\left(E_{k}^{-}+E_{k}^{+}\right)}, \\
& A_{12}=(-1) \frac{\Delta_{1 k}\left(E_{2 k}^{2}-\left(E_{k}^{+}\right)^{2}+\Delta_{2 k}^{2}\right)+\left(\Delta_{2 k}\right) \chi_{k}^{2}}{2 E_{k}^{+}\left(E_{k}^{-}-E_{k}^{+}\right)\left(E_{k}^{-}+E_{k}^{+}\right)}, \\
& A_{13}=\frac{\Delta_{2 k}\left(E_{1 k}^{2}-\left(E_{k}^{-}\right)^{2}+\Delta_{1 k}^{2}\right)+\Delta_{1 k} \chi_{k}^{2}}{2 E_{k}^{-}\left(E_{k}^{-}-E_{k}^{+}\right)\left(E_{k}^{-}+E_{k}^{+}\right)}, \\
& A_{14}=\frac{\Delta_{2 k}\left(E_{1 k}^{2}-\left(E_{k}^{-}\right)^{2}+\Delta_{1 k}^{2}\right)+\Delta_{1 k} \chi_{k}^{2}}{2 E_{k}^{-}\left(E_{k}^{-}-E_{k}^{+}\right)\left(E_{k}^{-}+E_{k}^{+}\right)}, \\
& A_{15}=(-1) \frac{\Delta_{2 k}\left(E_{1 k}^{2}-\left(E_{k}^{+}\right)^{2}+\Delta_{1 k}^{2}\right)+\left(\Delta_{1 k}\right) \chi_{k}^{2}}{2 E_{k}^{+}\left(E_{k}^{-}-E_{k}^{+}\right)\left(E_{k}^{-}+E_{k}^{+}\right)} \text {, } \\
& A_{16}=(-1) \frac{\Delta_{2 k}\left(E_{1 k}^{2}-\left(E_{k}^{+}\right)^{2}+\Delta_{1 k}^{2}\right)+\left(\Delta_{1 k}\right) \chi_{k}^{2}}{2 E_{k}^{+}\left(E_{k}^{-}-E_{k}^{+}\right)\left(E_{k}^{-}+E_{k}^{+}\right)} \text {, }
\end{aligned}
$$




$$
\begin{aligned}
& A_{17}=\frac{\chi_{k}\left[\left(E_{1 k}-E_{k}^{-}\right)\left(-E_{2 k}+E_{k}^{-}\right)+\Delta_{1 k} \Delta_{2 k}+\chi_{k}^{2}\right]}{2 E_{k}^{-}\left(E_{k}^{-}-E_{k}^{+}\right)\left(E_{k}^{-}+E_{k}^{+}\right)}, \\
& A_{18}=\frac{\chi_{k}\left[-\left(E_{1 k}+E_{k}^{-}\right)\left(E_{2 k}+E_{k}^{-}\right)+\Delta_{1 k} \Delta_{2 k}+\chi_{k}^{2}\right]}{2 E_{k}^{-}\left(E_{k}^{-}-E_{k}^{+}\right)\left(E_{k}^{-}+E_{k}^{+}\right)}, \\
& A_{19}=(-1) \frac{\chi_{k}\left[\left(E_{1 k}-E_{k}^{+}\right)\left(-E_{2 k}+E_{k}^{+}\right)+\Delta_{1 k} \Delta_{2 k}+\chi_{k}^{2}\right]}{2 E_{k}^{+}\left(E_{k}^{-}-E_{k}^{+}\right)\left(E_{k}^{-}+E_{k}^{+}\right)}, \\
& A_{20}=\frac{\chi_{k}\left[\left(E_{1 k}+E_{k}^{+}\right)\left(E_{2 k}+E_{k}^{+}\right)-\Delta_{1 k} \Delta_{2 k}-\chi_{k}^{2}\right]}{2 E_{k}^{+}\left(E_{k}^{-}-E_{k}^{+}\right)\left(E_{k}^{-}+E_{k}^{+}\right)}, \\
& A_{21}=A_{17}, \quad A_{22}=A_{18}, \quad A_{23}=A_{19}, \quad A_{24}=A_{20}, \\
& A_{25}=(-1) \frac{\chi_{k}\left[\left(E_{2 k}+E_{k}^{-}\right)\left(\Delta_{1 k}\right)+E_{1 k} \Delta_{2 k}-E_{k}^{-} \Delta_{2 k}\right]}{2 E_{k}^{-}\left(E_{k}^{-}-E_{k}^{+}\right)\left(E_{k}^{-}+E_{k}^{+}\right)}, \\
& A_{26}=(-1) \frac{\chi_{k}\left[\left(E_{2 k}-E_{k}^{-}\right)\left(\Delta_{1 k}\right)+E_{1 k} \Delta_{2 k}+E_{k}^{-} \Delta_{2 k}\right]}{2 E_{k}^{-}\left(E_{k}^{-}-E_{k}^{+}\right)\left(E_{k}^{-}+E_{k}^{+}\right)}, \\
& A_{27}=\frac{\chi_{k}\left[\left(E_{2 k}+E_{k}^{+}\right)\left(\Delta_{1 k}\right)+E_{1 k} \Delta_{2 k}-E_{k}^{+} \Delta_{2 k}\right]}{2 E_{k}^{+}\left(E_{k}^{-}-E_{k}^{+}\right)\left(E_{k}^{-}+E_{k}^{+}\right)}, \\
& A_{28}=\frac{\chi_{k}\left[\left(E_{2 k}-E_{k}^{+}\right)\left(\Delta_{1 k}\right)+E_{1 k} \Delta_{2 k}+E_{k}^{+} \Delta_{2 k}\right]}{2 E_{k}^{+}\left(E_{k}^{-}-E_{k}^{+}\right)\left(E_{k}^{-}+E_{k}^{+}\right)}, \\
& A_{29}=(-1) \frac{\chi_{k}\left[\left(E_{2 k}+E_{k}^{-}\right)\left(\Delta_{1 k}\right)+E_{1 k} \Delta_{2 k}-E_{k}^{-} \Delta_{2 k}\right]}{2 E_{k}^{-}\left(E_{k}^{-}-E_{k}^{+}\right)\left(E_{k}^{-}+E_{k}^{+}\right)}, \\
& A_{30}=(-1) \frac{\chi_{k}\left[\left(E_{2 k}-E_{k}^{-}\right)\left(\Delta_{1 k}\right)+E_{1 k} \Delta_{2 k}+E_{k}^{-} \Delta_{2 k}\right]}{2 E_{k}^{-}\left(E_{k}^{-}-E_{k}^{+}\right)\left(E_{k}^{-}+E_{k}^{+}\right)}, \\
& 2 E_{k}^{+}\left(E_{k}^{-}-E_{k}^{+}\right)\left(E_{k}^{-}+E_{k}^{+}\right)
\end{aligned}
$$

To evaluate the self-energies ( $\Pi$ ) in Eq. (B2), we first perform the summation over the Matsubara frequency using analytic tool of contour integration. Next we do the summation over $k$ using numerical tools taking $\Delta_{k}=\Delta_{k+Q}, g=1$, and $N=40000$. The plot of the self-energy in this case is presented in Fig. 3.

\section{APPENDIX C: CALCULATION OF SELF-ENERGY ח IN THE PRESENCE OF INVERSE LIFETIME $\Gamma$}

In this Appendix we present the self-energy calculation in the presence of finite inverse lifetime $(\Gamma)$ of the quasiparticles, associated to thermal fluctuations. Here Green's function elements become

$$
G_{i, j}\left(i \omega_{n}, k\right) \rightarrow G_{i, j}\left[i \omega_{n}+i \Gamma \operatorname{sgn}\left(\omega_{n}\right), k\right] .
$$

The self-energies $\Pi$ in Eq. (B2) now have the following general structure:

$$
\sum_{k, i \omega_{n}} G_{k}^{a}\left[i \omega_{n}+i \Gamma \operatorname{sgn}\left(\omega_{n}\right)\right] G_{k+q}^{b}\left[i \omega_{n}+i \Gamma \operatorname{sgn}\left(\omega_{n}\right)+i \epsilon_{n}\right],
$$

where either a or b symbolically represent the $(i, j)$ th element of the Green's function matrix. To evaluate the Matsubara summation in this case, we need to use a contour avoiding the branch cuts defined by $\operatorname{Im}(z)=0$ and $\operatorname{Im}\left(z+i \epsilon_{n}\right)=0$, as shown in the Fig. 7. Using this contour, we arrive at the following integrations:

$$
\begin{aligned}
I_{\gamma_{1}}= & \sum_{k}\left[\int_{-\infty}^{\infty} \frac{d \omega}{2 \pi i} n_{F}(\omega) G_{k}^{a}(\omega+i \Gamma) G_{k+q}^{b}(\omega+\epsilon+i \Gamma)\right], \\
I_{\gamma_{3}}= & -\sum_{k}\left[\int_{-\infty}^{\infty} \frac{d \omega}{2 \pi i} n_{F}(\omega) G_{k}^{a}(\omega-\epsilon-i \Gamma) G_{k+q}^{b}(\omega-i \Gamma)\right], \\
I_{\gamma_{2}}= & \sum_{k}\left[\int _ { - \infty } ^ { \infty } \frac { d \omega } { 2 \pi i } n _ { F } ( \omega ) \left(G_{k}^{a}(\omega-\epsilon-i \Gamma) G_{k+q}^{b}(\omega+i \Gamma)\right.\right. \\
& \left.\left.-G_{k}^{a}(\omega-i \Gamma) G_{k+q}^{b}(\omega+\epsilon+i \Gamma)\right)\right],
\end{aligned}
$$

where $n_{F}(\omega)=1 /(\exp \beta \omega+1)$ is the Fermi distribution function. Moreover, $\beta=1 / k_{B} T$, where $k_{B}$ is the Boltzmann constant. Next, in the limit $T \rightarrow 0$, the integrals $I_{\gamma_{1}}$ and $I_{\gamma_{3}}$ in Eq. (C3) become

$$
I_{\gamma_{1}}=\sum_{k}\left[\int_{-\infty}^{0} \frac{d \omega}{2 \pi i} G_{k}^{a}(\omega+i \Gamma) G_{k+q}^{b}(\omega+\epsilon+i \Gamma)\right]
$$




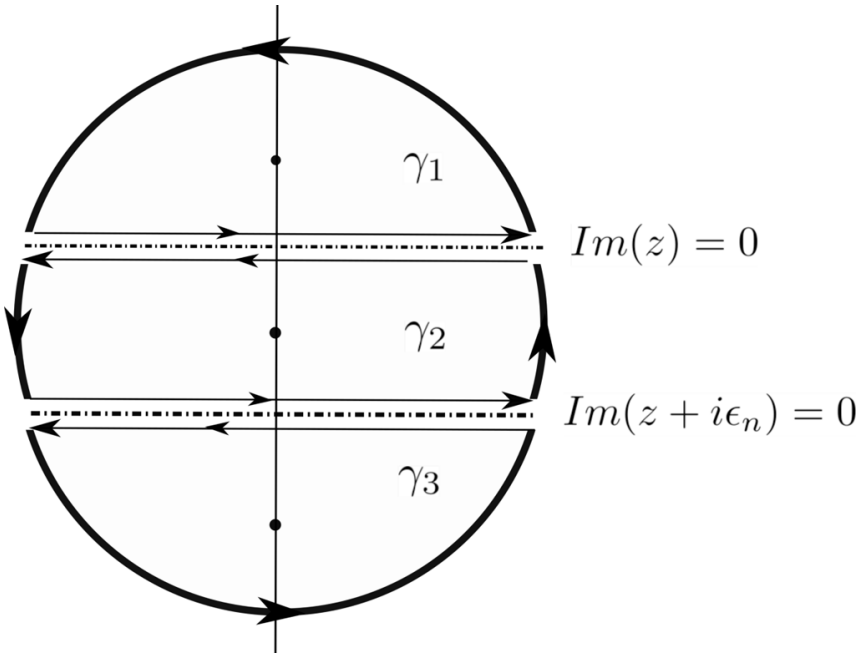

FIG. 7. Contour for complex Matsubara frequency summation: $\operatorname{Im}(z)=0$ and $\operatorname{Im}\left(z+i \epsilon_{n}\right)=0$ denote the two branch cuts in the complex plane. $\gamma_{1}, \gamma_{2}$, and $\gamma_{3}$ are the three contours of integration.

$$
I_{\gamma_{3}}=-\sum_{k}\left[\int_{-\infty}^{0} \frac{d \omega}{2 \pi i} G_{k}^{a}(\omega-\epsilon-i \Gamma) G_{k+q}^{b}(\omega-i \Gamma)\right] .
$$

We replace $\omega \rightarrow \omega+\epsilon$ in the first term of $I_{\gamma_{2}}$ and successively use

$$
\lim _{\epsilon \rightarrow 0} \frac{n_{F}(\omega+\epsilon)-n_{F}(\omega)}{\epsilon}=-\delta(\omega),
$$

where $\delta(\omega)$ is a Dirac $\delta$ function with the property $\int_{-\infty}^{\infty} d \omega f(\omega) \delta(\omega)=f(0)$. Thus $I_{\gamma_{2}}$ in Eq. (C3) becomes

$$
I_{\gamma_{2}}=\sum_{k} \frac{-\epsilon}{2 \pi i}\left[G_{k}^{a}(i \Gamma) G_{k+q}^{b}(\epsilon+i \Gamma)\right]
$$

Finally, we evaluate the real frequency $(\omega)$ integration in Eq. (C4) for each of the four self-energies $\Pi_{1}, \Pi_{2}, \Pi_{3}$, and $\Pi_{4}$ in Eq. (B2) by using

$$
\int_{-\infty}^{0} d \omega\left[\frac{1}{(\omega-x) *(\omega-y)}\right]=\frac{\log [x]-\log [y]}{x-y},
$$

where $x, y \in \mathbb{C}$. The summation over $k$ is again evaluated using numerical tools. The re-normalization of the phonon spectrum in this case is given by the real part of the self-energy. The real part of the self-energy is plotted for different sets of SC order, $\Gamma$, and $\mathrm{CDW}$ order in Figs. 4 and 5 .
[1] H. Alloul, T. Ohno, and P. Mendels, ${ }^{89} \mathrm{Y}$ NMR Evidence for a Fermi-Liquid Behavior in $\mathrm{YBa}_{2} \mathrm{Cu}_{3} \mathrm{O}_{6+\mathrm{x}}$, Phys. Rev. Lett. 63, 1700 (1989).

[2] W. W. Warren, R. E. Walstedt, G. F. Brennert, R. J. Cava, R. Tycko, R. F. Bell, and G. Dabbagh, Cu Spin Dynamics and Superconducting Precursor Effects in Planes Above $T_{c}$ in $\mathrm{YBa}_{2} \mathrm{Cu}_{3} \mathrm{O}_{6.7}$, Phys. Rev. Lett. 62, 1193 (1989).

[3] C. Berthier, M. H. Julien, M. Horvatić, and Y. Berthier, NMR studies of the normal state of high temperature superconductors, J. Phys. I France 6, 2205 (1996).

[4] D. S. Marshall, D. S. Dessau, A. G. Loeser, C.-H. Park, A. Y. Matsuura, J. N. Eckstein, I. Bozovic, P. Fournier, A. Kapitulnik, W. E. Spicer, and Z.-X. Shen, Unconventional Electronic Structure Evolution with Hole Doping in $\mathrm{Bi}_{2} \mathrm{Sr}_{2} \mathrm{CaCu}_{2} \mathrm{O}_{8+\delta}$ : Angle-Resolved Photoemission Results, Phys. Rev. Lett. 76, 4841 (1996).

[5] J. M. Harris, P. J. White, Z.-X. Shen, H. Ikeda, R. Yoshizaki, H. Eisaki, S. Uchida, W. D. Si, J. W. Xiong, Z.-X. Zhao, and D. S. Dessau, Measurement of an Anisotropic Energy Gap in Single Plane $\mathrm{Bi}_{2} \mathrm{Sr}_{2-x} \mathrm{La}_{x} \mathrm{CuO}_{6+\delta}$, Phys. Rev. Lett. 79, 143 (1997).

[6] Ch. Renner, B. Revaz, J.-Y. Genoud, K. Kadowaki, and Ø. Fischer, Pseudogap Precursor of the Superconducting Gap in Under- and Overdoped $\mathrm{Bi}_{2} \mathrm{Sr}_{2} \mathrm{CaCu}_{2} \mathrm{O}_{8+\delta}$, Phys. Rev. Lett. 80, 149 (1998).

[7] A. Ino, T. Mizokawa, K. Kobayashi, A. Fujimori, T. Sasagawa, T. Kimura, K. Kishio, K. Tamasaku, H. Eisaki, and S. Uchida, Doping Dependent Density of States and Pseudogap Behavior in $\mathrm{La}_{2-x} \mathrm{Sr}_{x} \mathrm{CuO}_{4}$, Phys. Rev. Lett. 81, 2124 (1998).

[8] F. Ronning, T. Sasagawa, Y. Kohsaka, K. M. Shen, A. Damascelli, C. Kim, T. Yoshida, N. P. Armitage, D. H. Lu, D. L.
Feng, L. L. Miller, H. Takagi, and Z.-X. Shen, Evolution of a metal to insulator transition in $\mathrm{Ca}_{2-x} \mathrm{Na}_{x} \mathrm{CuO}_{2} \mathrm{Cl}_{2}$ as seen by angle-resolved photoemission, Phys. Rev. B 67, 165101 (2003).

[9] M. R. Norman, H. Ding, M. Randeria, J. C. Campuzano, Takayoshi Yokoya, T. Takeuchi, T. Takahashi, T. Mochiku, K. Kadowaki, P. Guptasarma et al., Destruction of the Fermi surface in underdoped high-Tc superconductors, Nature (London) 392, 157 (1998).

[10] M. R. Norman and C. Pépin, The electronic nature of high temperature cuprate superconductors, Rep. Prog. Phys. 66, 1547 (2003).

[11] E. Fradkin, S. A. Kivelson, and J. M. Tranquada, Colloquium: Theory of intertwined orders in high temperature superconductors, Rev. Mod. Phys. 87, 457 (2015).

[12] C. Pépin, D. Chakraborty, M. Grandadam, and S. Sarkar, Fluctuations and the Higgs mechanism in underdoped cuprates, Annu. Rev. Condens. Matter Phys. 11, 301 (2020).

[13] J. E. Hoffman, E. W. Hudson, K. M. Lang, V. Madhavan, H. Eisaki, S. Uchida, and J. C. Davis, A four unit cell periodic pattern of quasi-particle states surrounding vortex cores in $\mathrm{Bi}_{2} \mathrm{Sr}_{2} \mathrm{CaCu}_{2} \mathrm{O}_{8+\delta}$, Science 295, 466 (2002).

[14] N. Doiron-Leyraud, C. Proust, D. LeBoeuf, J. Levallois, J.-B. Bonnemaison, R. Liang, D. A. Bonn, W. N. Hardy, and L. Taillefer, Quantum oscillations and the Fermi surface in an underdoped high- $T_{c}$ superconductor, Nature (London) 447, 565 (2007).

[15] G. Ghiringhelli, M. Le Tacon, M. Minola, S. Blanco-Canosa, C. Mazzoli, N. B. Brookes, G. M. De Luca, A. Frano, D. G. Hawthorn, F. He, T. Loew, M. Moretti Sala, D. C. Peets, M. Salluzzo, E. Schierle, R. Sutarto, G. A. Sawatzky, E. Weschke, B. Keimer, and L. Braicovich, Long-range incommensurate 
charge fluctuations in (Y, Nd) $\mathrm{Ba}_{2} \mathrm{Cu}_{3} \mathrm{O}_{6+x}$, Science 337, 821 (2012).

[16] H.-H. Wu, M. Buchholz, C. Trabant, C. F. Chang, A. C. Komarek, F. Heigl, M. V. Zimmermann, M. Cwik, F. Nakamura, M. Braden et al., Charge stripe order near the surface of 12-percent doped $\mathrm{La}_{2-x} \mathrm{Sr}_{x} \mathrm{CuO}_{4}$, Nat. Commun. 3, 1023 (2012).

[17] A. J. Achkar, R. Sutarto, X. Mao, F. He, A. Frano, S. BlancoCanosa, M. Le Tacon, G. Ghiringhelli, L. Braicovich, M. Minola, M. Moretti Sala, C. Mazzoli, Ruixing Liang, D. A. Bonn, W. N. Hardy, B. Keimer, G. A. Sawatzky, and D. G. Hawthorn, Distinct Charge Orders in the Planes and Chains of Ortho-iii-Ordered $\mathrm{YBa}_{2} \mathrm{Cu}_{3} \mathbf{O}_{6+\delta}$ Superconductors Identified by Resonant Elastic X-Ray Scattering, Phys. Rev. Lett. 109, 167001 (2012).

[18] E. Blackburn, J. Chang, M. Hücker, A. T. Holmes, N. B. Christensen, Ruixing Liang, D. A. Bonn, W. N. Hardy, U. Rütt, O. Gutowski, M. v. Zimmermann, E. M. Forgan, and S. M. Hayden, X-Ray Diffraction Observations of a Charge-DensityWave Order in Superconducting Ortho-ii $\mathrm{YBa}_{2} \mathrm{Cu}_{3} \mathbf{O}_{6.54}$ Single Crystals in Zero Magnetic Field, Phys. Rev. Lett. 110, 137004 (2013).

[19] E. Blackburn, J. Chang, A. H. Said, B. M. Leu, Ruixing Liang, D. A. Bonn, W. N. Hardy, E. M. Forgan, and S. M. Hayden, Inelastic $\mathrm{x}$-ray study of phonon broadening and charge-density wave formation in ortho-ii-ordered $\mathrm{yba}_{2} \mathrm{Cu}_{3} \mathrm{O}_{6.54}$, Phys. Rev. B 88, 054506 (2013).

[20] S. Blanco-Canosa, A. Frano, T. Loew, Y. Lu, J. Porras, G. Ghiringhelli, M. Minola, C. Mazzoli, L. Braicovich, E. Schierle, E. Weschke, M. Le Tacon, and B. Keimer, MomentumDependent Charge Correlations in $\mathrm{YBa}_{2} \mathrm{Cu}_{3} \mathrm{O}_{6+\delta}$ Superconductors Probed by Resonant X-Ray Scattering: Evidence for Three Competing Phases, Phys. Rev. Lett. 110, 187001 (2013).

[21] T. P. Croft, C. Lester, M. S. Senn, A. Bombardi, and S. M. Hayden, Charge density wave fluctuations in $\mathrm{La}_{2-x} \mathrm{Sr}_{x} \mathrm{CuO}_{4}$ and their competition with superconductivity, Phys. Rev. B 89, 224513 (2014).

[22] E. H. da Silva Neto, P. Aynajian, A. Frano, R. Comin, E. Schierle, E. Weschke, A. Gyenis, J. Wen, J. Schneeloch, Z. $\mathrm{Xu}, \mathrm{S}$. Ono, G. Gu, M. Le Tacon, and A. Yazdani, Ubiquitous interplay between charge ordering and high-temperature superconductivity in cuprates, Science 343, 393 (2014).

[23] K. Matsuba, S. Yoshizawa, Y. Mochizuki, T. Mochiku, K. Hirata, and N. Nishida, Anti-phase modulation of electron-and hole-like states in vortex core of $\mathrm{Bi}_{2} \mathrm{Sr}_{2} \mathrm{CaCu}_{2} \mathrm{O}_{x}$ probed by scanning tunneling spectroscopy, J. Phys. Soc. Jpn. 76, 063704 (2007).

[24] K. Fujita, C. K. Kim, I. Lee, J. Lee, M. H. Hamidian, I. A. Firmo, S. Mukhopadhyay, H. Eisaki, S. Uchida, M. J. Lawler, E. A. Kim, and J. C. Davis, Simultaneous transitions in cuprate momentum-space topology and electronic symmetry breaking, Science 344, 612 (2014).

[25] T. Machida, Y. Kohsaka, K. Matsuoka, K. Iwaya, T. Hanaguri, and T. Tamegai, Bipartite electronic superstructures in the vortex core of $\mathrm{Bi}_{2} \mathrm{Sr}_{2} \mathrm{CaCu}_{2} \mathrm{O}_{8+\delta}$, Nat. Commun. 7, 11747 (2016).

[26] W. D. Wise, M. C. Boyer, K. Chatterjee, T. Kondo, T. Takeuchi, H. Ikuta, Y. Wang, and E. W. Hudson, Charge-density-wave origin of cuprate checkerboard visualized by scanning tunneling microscopy, Nat. Phys. 4, 696 (2008).

[27] E. J. Woll and W. Kohn, Images of the Fermi surface in phonon spectra of metals, Phys. Rev. 126, 1693 (1962).

[28] B. Renker, H. Rietschel, L. Pintschovius, W. Gläser, P. Brüesch, D. Kuse, and M. J. Rice, Observation of Giant Kohn Anomaly in the One-Dimensional Conductor $\mathrm{K}_{2} \mathrm{Pt}(\mathrm{CN})_{4} \mathrm{Br}_{0: 3} \cdot 3 \mathrm{H}_{2} O$, Phys. Rev. Lett. 30, 1144 (1973).

[29] K. Carneiro, G. Shirane, S. A. Werner, and S. Kaiser, Lattice dynamics of $\mathrm{K}_{2} \mathrm{Pt}(\mathrm{CN})_{4} \mathrm{Br}_{0: 3} \cdot 3 \cdot 2 \mathrm{D}_{2} \mathrm{O}(K C P)$ studied by inelastic neutron scattering, Phys. Rev. B 13, 4258 (1976).

[30] J. P. Pouget, B. Hennion, C. Escribe-Filippini, and M. Sato, Neutron-scattering investigations of the kohn anomaly and of the phase and amplitude charge-density-wave excitations of the blue bronze $\mathrm{K}_{0.3} \mathrm{MoO}_{3}$, Phys. Rev. B 43, 8421 (1991).

[31] J. A. Wilson, F. J. Di Salvo, and S. Mahajan, Charge-density waves and superlattices in the metallic layered transition metal dichalcogenides, Adv. Phys. 50, 1171 (2001).

[32] M. Le Tacon, A. Bosak, S. M. Souliou, G. Dellea, T. Loew, R. Heid, K.-P. Bohnen, G. Ghiringhelli, M. Krisch, and B. Keimer, Inelastic $\mathrm{x}$-ray scattering in $\mathrm{YBa}_{2} \mathrm{Cu}_{3} \mathrm{O}_{6.6}$ reveals giant phonon anomalies and elastic central peak due to charge-density-wave formation, Nat. Phys. 10, 52 (2014).

[33] H. Miao, D. Ishikawa, R. Heid, M. Le Tacon, G. Fabbris, D. Meyers, G. D. Gu, A. Q. R. Baron, and M. P. M. Dean, Incommensurate Phonon Anomaly and the Nature of Charge Density Waves in Cuprates, Phys. Rev. X 8, 011008 (2018).

[34] W. S. Lee, K. J. Zhou, M. Hepting, J. Li, A. Nag, A. C. Walters, M. Garcia-Fernandez, H. Robarts, M. Hashimoto, H. Lu et al., Spectroscopic evidence for charge order melting via quantum fluctuations in a cuprate, Nat. Phys. 17, 53 (2021).

[35] H. Uchiyama, A. Q. R. Baron, S. Tsutsui, Y. Tanaka, W.-Z. $\mathrm{Hu}, \mathrm{A}$. Yamamoto, S. Tajima, and Y. Endoh, Softening of $\mathrm{Cu}-\mathrm{o}$ Bond Stretching Phonons in Tetragonal $\mathrm{HgBa}_{2} \mathrm{CuO}_{4+\delta}$, Phys. Rev. Lett. 92, 197005 (2004).

[36] D. Reznik, T. Fukuda, D. Lamago, A. Q. R. Baron, S Tsutsui, M. Fujita, and K. Yamada, q-dependence of the giant bond-stretching phonon anomaly in the stripe compound $\mathrm{La}_{1.48} \mathrm{Nd}_{0.4} \mathrm{Sr}_{0.12} \mathrm{CuO}_{4}$ measured by IXS, J. Phys. Chem. Solids 69, 3103 (2008).

[37] J. Graf, M. d'Astuto, C. Jozwiak, D. R. Garcia, N. L. Saini, M. Krisch, K. Ikeuchi, A. Q. R. Baron, H. Eisaki, and A. Lanzara, Bond Stretching Phonon Softening and Kinks in the Angle-Resolved Photoemission Spectra of Optimally Doped $\mathrm{Bi}_{2} \mathrm{Sr}_{1.6} \mathrm{La}_{0.4} \mathrm{Cu}_{2} \mathrm{O}_{6+\delta}$ Superconductors, Phys. Rev. Lett. 100, 227002 (2008).

[38] M. d'Astuto, G. Dhalenne, J. Graf, M. Hoesch, P. Giura, M. Krisch, P. Berthet, A. Lanzara, and A. Shukla, Sharp opticalphonon softening near optimal doping in $\mathrm{La}_{2-x} \mathrm{Ba}_{x} \mathrm{CuO}_{4+\delta}$ observed via inelastic x-ray scattering, Phys. Rev. B 78, 140511(R) (2008).

[39] A. Q. R. Baron, J. P. Sutter, S. Tsutsui, H. Uchiyama, T. Masui, S. Tajima, R. Heid, and K.-P. Bohnen, First study of the $\mathrm{b} 1 \mathrm{~g}$ buckling phonon mode in optimally doped, de-twinned, $\mathrm{YBa}_{2} \mathrm{Cu}_{3} \mathrm{O}_{7-\delta}$ by inelastic $\mathrm{x}$-ray scattering, J. Phys. Chem. Solids 69, 3100 (2008).

[40] R. J. McQueeney, Y. Petrov, T. Egami, M. Yethiraj, G. Shirane, and Y. Endoh, Anomalous Dispersion of LO Phonons in 
$\mathrm{La}_{1.85} \mathrm{Sr}_{0.15} \mathrm{CuO}_{4}$ at Low Temperatures, Phys. Rev. Lett. 82, 628 (1999).

[41] M. Raichle, D. Reznik, D. Lamago, R. Heid, Y. Li, M. Bakr, C. Ulrich, V. Hinkov, K. Hradil, C. T. Lin, and B. Keimer, Highly Anisotropic Anomaly in the Dispersion of the Copper-Oxygen Bond-Bending Phonon in Superconducting $\mathrm{YBa}_{2} \mathrm{Cu}_{3} \mathrm{O}_{7}$ from Inelastic Neutron Scattering, Phys. Rev. Lett. 107, 177004 (2011).

[42] J. E. Lorenzo, R. Currat, P. Monceau, B. Hennion, H. Berger, and F. Levy, A neutron scattering study of the quasi-onedimensional conductor, J. Phys.: Condens. Matter 10, 5039 (1998).

[43] H. Requardt, J. E. Lorenzo, P. Monceau, R. Currat, and M. Krisch, Dynamics in the charge-density-wave system $\mathrm{NbSe}_{3}$ using inelastic $\mathrm{x}$-ray scattering with $\mathrm{meV}$ energy resolution, Phys. Rev. B 66, 214303 (2002).

[44] M. A. Metlitski and S. Sachdev, Quantum phase transitions of metals in two spatial dimensions, I. Ising-nematic order, Phys. Rev. B 82, 075127 (2010).

[45] K. B. Efetov, H. Meier, and C. Pépin, Pseudogap state near a quantum critical point, Nat. Phys. 9, 442 (2013).

[46] L. E. Hayward, D. G. Hawthorn, R. G. Melko, and S. Sachdev, Angular Fluctuations of a Multicomponent Order Describe the Pseudogap of $\mathrm{YBa}_{2} \mathrm{Cu}_{3} \mathrm{O}_{6+x}$, Science 343, 1336 (2014).

[47] Y. Wang and A. Chubukov, Charge-density-wave order with momentum $(2 q, 0)$ and $(0,2 q)$ within the spin-fermion model: Continuous and discrete symmetry breaking, preemptive composite order, and relation to pseudogap in hole-doped cuprates, Phys. Rev. B 90, 035149 (2014).

[48] Y. Wang, D. F. Agterberg, and A. Chubukov, Coexistence of Charge-Density-Wave and Pair-Density-Wave Orders in Underdoped Cuprates, Phys. Rev. Lett. 114, 197001 (2015).

[49] D. Chakraborty, C. Morice, and C. Pépin, Phase diagram of the underdoped cuprates at high magnetic field, Phys. Rev. B 97, 214501 (2018).

[50] B. Loret, N. Auvray, Y. Gallais, M. Cazayous, A. Forget, D. Colson, M.-H. Julien, I. Paul, M. Civelli, and A. Sacuto, Intimate link between charge density wave, pseudogap and superconducting energy scales in cuprates, Nat. Phys. 15, 771 (2019).

[51] J. Chang, E. Blackburn, A. T. Holmes, N. B. Christensen, J. Larsen, J. Mesot, Ruixing Liang, D. A. Bonn, W. N. Hardy, A. Watenphul, M. v. Zimmermann, E. M. Forgan, and S. M. Hayden, Direct observation of competition between superconductivity and charge density wave order in $\mathrm{YBa}_{2} \mathrm{Cu}_{3} \mathrm{O}_{6.67}$, Nat. Phys. 8, 871 (2012).

[52] D. Chakraborty, M. Grandadam, M. H. Hamidian, J. C. S. Davis, Y. Sidis, and C. Pépin, Fractionalized pair density wave in the pseudogap phase of cuprate superconductors, Phys. Rev. B 100, 224511 (2019).

[53] S. D. Edkins, A. Kostin, K. Fujita, A. P. Mackenzie, Hiroshi Eisaki, S. Uchida, Subir Sachdev, M. J. Lawler, E.-A. Kim, J. C. Séamus Davis et al., Magnetic field-induced pair density wave state in the cuprate vortex halo, Science 364, 976 (2019).

[54] M. H. Hamidian, S. D. Edkins, S. H. Joo, A. Kostin, H. Eisaki, S. Uchida, M. J. Lawler, E.-A. Kim, A. P. Mackenzie, and K. Fujita et al., Detection of a cooper-pair density wave in $\mathrm{Bi}_{2} \mathrm{Sr}_{2} \mathrm{CaCu}_{2} \mathrm{O}_{8+x}$, Nature (London) 532, 343 (2016).
[55] M. Grandadam, D. Chakraborty, X. Montiel, and C. Pépin, Electronic spectral function in the fractionalized pair density wave scenario, Phys. Rev. B 102, 121104(R) (2020).

[56] M. R. Norman, M. Randeria, H. Ding, and J. C. Campuzano, Phenomenology of the low-energy spectral function in high- $T_{c}$ superconductors, Phys. Rev. B 57, R11093 (1998).

[57] P. A. Lee, T. M. Rice, and P. W. Anderson, Conductivity from charge or spin density waves, Solid State Commun. 88, 1001 (1993).

[58] C. Berthod, I. Maggio-Aprile, J. Bruér, A. Erb, and C. Renner, Observation of Caroli-de Gennes-Matricon Vortex States in $\mathrm{YBa}_{2} \mathrm{Cu}_{3} \mathrm{O}_{7-\delta}$, Phys. Rev. Lett. 119, 237001 (2017).

[59] D. Chowdhury and S. Sachdev, Feedback of superconducting fluctuations on charge order in the underdoped cuprates, Phys. Rev. B 90, 134516 (2014).

[60] R. Comin, A. Frano, M. M. Yee, Y. Yoshida, H. Eisaki, E. Schierle, E. Weschke, R. Sutarto, F. He, A. Soumyanarayanan, Yang He, M. Le Tacon, I. S. Elfimov, Jennifer E. Hoffman, G. A. Sawatzky, B. Keimer, and A. Damascelli, Charge order driven by Fermi-arc instability in $\mathrm{Bi}_{2} \mathrm{Sr}_{2-x} \mathrm{La}_{x} \mathrm{Cu} 0_{6}$, Science 343, 390 (2014).

[61] D. J. Scalapino, The case for $d_{x^{2}-y^{2}}$ pairing in the cuprate superconductors, Phys. Rep. 250, 329 (1995).

[62] M. R. Norman, M. Randeria, H. Ding, and J. C. Campuzano, Phenomenological models for the gap anisotropy of $\mathrm{Bi}_{2} \mathrm{Sr}_{2} \mathrm{CaCu}_{2} \mathrm{O}_{8}$ as measured by angle-resolved photoemission spectroscopy, Phys. Rev. B 52, 615 (1995).

[63] M. R. Norman, A. Kanigel, M. Randeria, U. Chatterjee, and J. C. Campuzano, Modeling the Fermi arc in underdoped cuprates, Phys. Rev. B 76, 174501 (2007).

[64] E. G. Dalla Torre, Y. He, D. Benjamin, and Eugene Demler, Exploring quasiparticles in high-Tc cuprates through photoemission, tunneling, and $\mathrm{x}$-ray scattering experiments, New J. Phys. 17, 022001 (2015).

[65] J. D. Axe and G. Shirane, Inelastic-neutron-scattering study of acoustic phonons in $\mathrm{Nb}_{3} \mathrm{Sn}$, Phys. Rev. B 8, 1965 (1973).

[66] D. Reznik, Phonon anomalies and dynamic stripes, Physica C: Superconductivity 481, 75 (2012).

[67] B. Loret, N. Auvray, G. D. Gu, A. Forget, D. Colson, M Cazayous, Y. Gallais, I. Paul, M. Civelli, and A. Sacuto, Universal relationship between the energy scales of the pseudogap phase, the superconducting state, and the charge-density-wave order in copper oxide superconductors, Phys. Rev. B 101, 214520 (2020).

[68] A. V. Chubukov, M. R. Norman, A. J. Millis, and E. Abrahams, Gapless pairing and the Fermi arc in the cuprates, Phys. Rev. B 76, 180501(R) (2007).

[69] A. Kanigel, M. R. Norman, M. Randeria, U. Chatterjee, S. Souma, A. Kaminski, H. M. Fretwell, S. Rosenkranz, M. Shi, T. Sato et al., Evolution of the pseudogap from Fermi arcs to the nodal liquid, Nat. Phys. 2, 447 (2006).

[70] C. M. Varma, P. B. Littlewood, S. Schmitt-Rink, E. Abrahams, and A. E. Ruckenstein, Phenomenology of the Normal State of Cu-O High-Temperature Superconductors, Phys. Rev. Lett. 63, 1996 (1989). 
[71] J. Q. Lin, H. Miao, D. G. Mazzone, G. D. Gu, A. Nag, A. C. Walters, M. García-Fernández, A. Barbour, J. Pelliciari, I. Jarrige, M. Oda, K. Kurosawa, N. Momono, K.-J. Zhou, V. Bisogni, X. Liu, and M. P. M. Dean, Strongly Correlated Charge Density Wave in $\mathrm{La}_{2-x} \mathrm{Sr}_{x} \mathrm{CuO}_{4}$ Evidenced by Doping-
Dependent Phonon Anomaly, Phys. Rev. Lett. 124, 207005 (2020).

[72] R. Comin and A. Damascelli, Resonant x-ray scattering studies of charge order in cuprates, Annu. Rev. Condens. Matter Phys. 7, 369 (2016). 